\title{
Editorial
}

\section{Endoscopic Thyroid Surgery}

Minimally invasive and remote access thyroidectomy using endoscopy has gained in popularity for the treatment of thyroid tumors. It has been developed to minimize surgical morbidity and to avoid or hide the visible neck scarring that is one of the drawbacks of conventional trans-cervical thyroidectomy, because neck scarring is a major concern in thyroid surgery, especially in young women. Endoscopic via cervical, axillary, anterior chest, breast, postauricular or transoral approaches has been developed over the past 20 years and has been recently refined.

Gagner first described the endoscopic subtotal parathyroidectomy with constant CO2 gas insufflations for hyperparathyroidism in 1996 and achieved a good clinical and cosmetic result.Since then minimal access parathyroidectomy has found a role alongside conventional cervicotomy for the treatment of primary hyperparathyroidism.

Huscher and colleagues first described the complete endoscopic right thyroid lobectomy in 1997. Minimally invasive surgery using endoscopic vision is now widely employed for the treatment of thyroid diseases for cosmetic purposes. Since then several approaches to the thyroid have evolved including the cervical approach, the minimally invasive video-assisted thyroidectomy (MIVAT), the transaxillary approach and the breast or anterior chest wall approach. Each of these approaches have their own advantages and disadvantages.Videoscopic neck surgery is developing despite the fact that only potential spaces exist in the neck. These approaches are more appealing since the size of incision of that the conventional approach seems to be out of proportion compared to the small size of the tumors.
Endoscopic surgery has reduced the level of surgical "invasiveness" and results in an improved cosmetic appearance. The site of approach is the most important factor because there is an intimate relationship between the locations of the trocars in terms of the cosmetic result, invasiveness, safety and ease of use.

The cervical approach utilizes small incisions in the neck thus making it cosmetically unacceptable and cannot be used for lesions greater than $4 \mathrm{~cm}$. Only patients who have small nodules with a low index of suspected malignancy are offered this endosopic approach.The operative field is small and because the camera is near the anatomic structures, it often has to be removed for cleaning, which significantly increases the operating time.

The axillary approach makes it difficult to visualize the opposite lobe. Although sectioning the sternohyoid muscle creates a good visual space even for the contralateral region and enables the contra lateral gland of the thyroid to be resected, the operating time is extremely prolonged and the additional scar tissue causes discomfort while swallowing and neck pain as a result of adhesions. Therefore this endoscopic procedure is not indicated for thyroid nodules that extend to the contralateral thyroid lobe.

The anterior chest wall approach utilizes port access at various positions on the anterior chest wall depending on the surgeon, thus avoiding a cervical incision. the trocars are over the sternum and infraclavicularly. These are hidden by the clothes of the patient and are not visible routinely.

This technique also allows bilateral neck exploration. Hence have been able to perform total thyroidectomies with central 
compartment clearance for papillary carcinoma and near-total thyroidectomies for large multinodular goiters..

The surgeries performed are hemithyroidectomy, total thyroidectomy and near- total thyroidectomies.

In differentiated thyroid cancer, surgical treatment depends on the risk group of the patient. Low-risk group patients underwent ipsilateral lobectomy and high and intermediate risk group patients underwent near total / total thyroidectomy.

The technique is safe and effective in the hands of an appropriately trained surgeon. To minimize surgical morbidity and neck scarring, minimally invasive thyroidectomy and endoscopic thyroidectomy via cervical, axillary, anterior chest, breast, postauricular or transoral approaches have been developed over the past 20 years. Among remote access approaches, the gasless transaxillary approach, bilateral axillo-breast approach, postauricular facelift approach, and transoral vestibular approach are in common use today. Each procedure has its own advantages and disadvantages. Therefore, we need to understand these advantages and limitations, and to select the appropriate method for each patient. The most significant advantage of remote access thyroidectomy is its excellent cosmesis. The complication rate is similar in patients undergoing a remote access approach and those undergoing conventional surgery if the former is performed by experienced surgeons. Operative time is significantly longer in remote access thyroidectomy.

\section{Classification of Endoscopic Thyroidectomy}

Endoscopic thyroidectomy can be classified according to the use of carbon dioxide (CO2) gas insufflation and the site of incision.

\section{CO2 insufflation method}

Cervical approach; Anterior chest approach; Axillary approach; Breast approach with parasternal port; Axillo-breast approach ; Axillo-bilateral breast approach; Bilateral axillo-breast approach; Unilateral/bilateral axillo-breast approach; Transoral approach

\section{Gasless method}

Minimally invasive video-assisted thyroidectomy; Anterior chest approach; Video-assisted neck surgery; Axillary approach; Axillary approach with anterior chest port ;Single incision axillary approach Gasless unilateral axillo-breast or axillary approach.

Endoscopic thyroidectomy using a remote access approach is feasible and comparable to conventional transcervical thyroidectomy in highly selected patients; it also yields excellent cosmesis. However, it has disadvantages in terms of longer operative time, higher cost, and technical difficulty. Strict patient selection criteria are very important. We also need to understand the advantages and limitations of various types of remote access thyroidectomy.

- Minimally invasive video-assisted thyroidectomy, transaxillary, bilateral axillo-breast approach, facelift and transoral approaches are in common use today.

- Remote access thyroidectomy is feasible and comparable to conventional thyroidectomy.

- The most important advantage of remote access thyroidectomy is its excellent cosmesis.

- The various approaches have their own advantages and disadvantages.

- Strict patient selection criteria are important
Prof. Dr. Mahmudul Hassan
Professor \& Director (Ex)
National Institute of ENT.
Love road .Tejgaon industrial area.
Dhaka-1208. 